\title{
DIELECTRIC FUNCTION THEORY CALCULATIONS OF POLARITONS IN GaN
}

\author{
K.P. Korona, R. StĘPNiEwsKi and J.M. Baranowski
}

Institute of Experimental Physics, Warsaw University, Hoża 69, 00-681 Warszawa, Poland

Properties of polaritons (free excitons coupled with photons of similar energy) in gallium nitride are investigated by performing calculations based on dielectric function theory including all three excitons $A, B$ and $C$ (characteristic for the wurtzite structure). Moreover the excited states of excitons have been taken into account by adding Elliott's components to dielectric function. Energies, polarizabilities and damping constants of excitons are determined. It is shown that due to inter-exciton interactions the B and C excitons are strongly damped. It is estimated that the characteristic time of $B$ to $A$ relaxation is $t_{\mathrm{BA}}=1 \mathrm{ps}$. The exciton $\mathrm{C}$ lifetime is estimated $\tau_{\mathrm{C}}=0.2 \mathrm{ps}$.

PACS numbers: 71.36.+c, 78.66.Fd

Strong coupling of free excitons with photons of similar energy leads to creation of polaritons. This phenomenon has been studied in different materials. However in GaN there are some difficulties. At first due to the wurtzite structure the valence band is splitted into three branches of $\Gamma_{9}, \Gamma_{7}$ and $\Gamma_{7}$ symmetries. The resulting three excitons have energies closely spaced, the differences between the excitons $A$ and $B$, and between $B$ and $C$ are about $5 \mathrm{meV}$ and $17 \mathrm{meV}$, respectively [1]. Such a small separation (smaller than exciton binding energy) means that one exciton interferes with another. In order to properly describe the optical constants which determine the measured spectrum, different excitons cannot be treated separately, like in CdS [2], but coupling of an electromagnetic wave with the excitons $\mathrm{A}, \mathrm{B}$, and $\mathrm{C}$ has to be taken into account simultaneously. The second difficulty are wide line widths of the peaks, which suggests strong damping of polaritons. This fact can significantly change the shape of the polariton dispersion curves.

The following calculation procedure has been realised:

(1) Polariton dispersion curves have been calculated by solving the coupled equations [3]

$$
\begin{aligned}
& \frac{\partial^{2} P_{\mathrm{X}}}{\partial t^{2}}+\omega_{\mathrm{X}}^{2}(k) P_{\mathrm{X}}=\alpha_{\mathrm{X}} \omega_{\mathrm{X}}^{2}(k) E-\gamma_{\mathrm{X}} \frac{\partial P_{\mathrm{X}}}{\partial t}, \quad \mathrm{X}=\mathrm{A}, \mathrm{B}, \mathrm{C} \\
& \varepsilon^{*} \frac{\partial^{2} E}{\partial t^{2}}+c^{2} \Delta E=-4 \pi \frac{\partial^{2}\left(P_{\mathrm{A}}+P_{\mathrm{B}}+P_{\mathrm{C}}\right)}{\partial t^{2}}
\end{aligned}
$$


where $E$ is a photon electric field and $P_{\mathrm{X}}$ is a polarization contribution due to an exciton X. Equation (1) represents the excitonic oscillator coupled with a photon electric field through polarizability $\alpha_{\mathbf{X}}$, damped with the damping constant $\gamma_{\mathbf{X}}$. The $k$-dependent frequency of the oscillator, $\omega_{\mathrm{X}}(k)=E_{\mathrm{X}} / \hbar=\omega_{\mathrm{XT}}+\hbar k^{2} / 2 M$, includes the kinetic energy of the free exciton with the wave vector $k$ and mass $M=m_{\mathrm{e}}+m_{\mathrm{h}}$. Equation (2) describes the electromagnetic wave in a material with the residual dielectric constant $\varepsilon^{*}$. The influence of the excitonic excited states (discrete and continuous) has been included by adding to the dielectric constant $\varepsilon^{*}$ components calculated from Elliott's formulas [4]. The resulting polariton dispersion curves are plotted in Fig. 1.
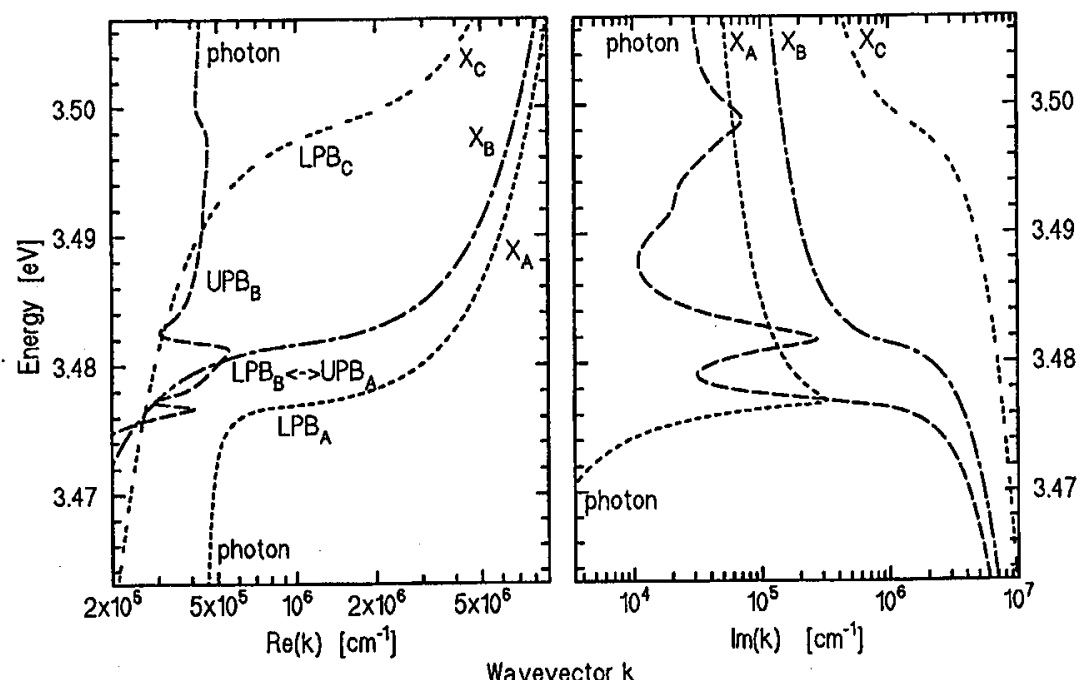

Fig. 1. The calculated polariton dispersion curves of GaN. The polariton branches arising from photon coupling with the excitons $A, B$ and $C$ are labelled: $L P B_{X}$ for the lower polariton branch and UPB $\mathrm{X}$ for the upper polariton branch of exciton $\mathrm{X}$.

(2) The effective dielectric function including all four polariton branches has been calculated. According to Pekar's additional boundary conditions [5], the polarizations of excitons vanishes on the surface $P_{\mathrm{A}}=P_{\mathrm{B}}=P_{\mathrm{C}}=0$. Moreover excitons are excluded from the surface layer of thickness $d$ (the so-called dead layer). As a consequence, the reflectivity is a function of two interfering waves with the phase difference $\Theta=2 d k n_{0}$ [6]. Taking this into account a reflectance spectrum has been calculated (Fig. 2).

(3) In the last step, the calculated reflectance spectrum was compared with experimental data measured on homoepitaxial GaN [1].

The points 1-3 has been repeated as long as a good fit to the experimental data has been obtained. The deduced parameters of individual excitons are listed in Table. The center of gravity mass $M$ has been assumed the same for all the excitons $M=1 m_{\mathrm{e}}$. Moreover from the fitting procedure the following parameters have been obtained: exciton binding energy (necessary for Elliott's dielectric function 


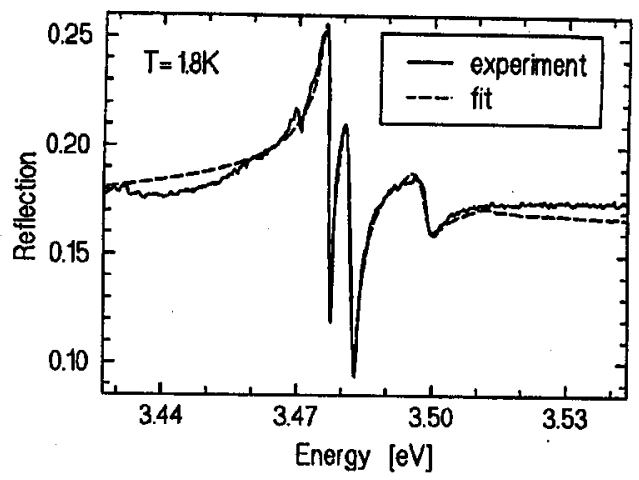

Fig. 2. Reflectance spectra of homoepitaxial GaN: solid curve - experiment [1], dashed curve - calculations.

\section{TABLE}

The obtained parameters of exciton polaritons in $\mathrm{GaN}$.

\begin{tabular}{c|c|c|c}
\hline \hline Exciton & $\begin{array}{c}\text { Energy } E_{\mathrm{T}}=\hbar \omega_{\mathrm{T}} \\
{[\mathrm{eV}]}\end{array}$ & Polarizability & $\begin{array}{c}\text { Damping constant } \\
{\left[10^{12} \mathrm{~s}^{-1}\right]}\end{array}$ \\
\hline $\mathrm{A}$ & 3.4767 & 0.0027 & 1.1 \\
$\mathrm{~B}$ & 3.4815 & 0.0031 & 2.3 \\
$\mathrm{C}$ & 3.4986 & 0.0011 & 4.7
\end{tabular}

calculations) $E_{\mathrm{G}}=20 \mathrm{meV}$, the background refractive index $n_{0}=2.4$, the dead layer thickness $d=4 \mathrm{~nm}$.

The dispersion curves are plotted in the scale of energy versus the real and imaginary parts of the wave vector $k$. The polariton branches arising from photon coupling with the exciton $\mathrm{X}$ are usually called: a lower polariton branch (LPBX) and an upper polariton branch (UPBX). In the case of three excitons $A, B$ and $C$ there are in fact four (not six) branches. In the case of strong coupling the UPB of lower exciton is merged with LPB of the higher one due to the anticrossing effect. In fact the $\mathrm{LPB}_{\mathrm{B}}$ and $\mathrm{UPB}_{\mathrm{A}}$ are merged in one branch. In the case of the exciton $\mathrm{C}$ the configuration is different. The coupling is so small (relatively to damping) that there is no anticrossing. Instead we observe one branch photon-like which for the high energies asymptotically tends to the photonic curve $(E=n \hbar k / c)$ and the other which is exciton-like $\left(E_{\mathrm{X}}=E_{\mathrm{XT}}+\hbar^{2} k^{2} / 2 M\right)$. For energies below excitons, there are also four polaritonic branches but only one (photon-like branch) has a small imaginary part. The other three curves have imaginary parts of the wave vectors $\operatorname{Im}(k)$ of the order of $10^{7} \mathrm{~cm}^{-1}$, which (comparing to wavelengths $\lambda$ of the order of $10^{-4} \mathrm{~cm}$ ) means that these are nonpropagating modes.

It is found that a damping constant of the exciton $A$ is the smallest one. The exciton $\mathrm{B}$ has a damping constant about twice higher. The polarizability of the exciton $B$ is similar to the exciton A, so the damping caused by scattering and recombination should be also similar. Therefore, the additional damping is probably 
connected with B to A relaxation process. The lower branch of the exciton B is merged with the upper branch of the exciton $A$ and the energy difference is only $5 \mathrm{meV}$ which makes the $\mathrm{B}$ to $\mathrm{A}$ relaxation very fast. The damping connected with this process can be estimated as $\gamma_{\mathrm{AB}}=\gamma_{\mathrm{B}}-\gamma_{\mathrm{A}}=10^{12} \mathrm{~s}^{-1}$. It means that the time of this process is about $t_{\mathrm{AB}}=1 \mathrm{ps}$. The strongest damping is observed in the case of exciton C. The obtained exciton binding energy $E_{\mathrm{G}}=20.2 \pm 0.3 \mathrm{meV}$ indicates that the exciton $\mathrm{C}$ is nearly degenerated with the excited states of the excitons $\mathrm{A}$ and $B$, which should cause a very fast decay of the exciton $C$. Its lifetime can be estimated as $\tau_{\mathrm{C}}=0.2 \mathrm{ps}$.

In summary, the energies, polarizabilities and damping constants of excitons are determined. It is shown that due to the inter-exciton interactions the excitons $\mathrm{B}$ and $\mathrm{C}$ are strongly damped. This effect significantly changes dispersion curves especially in the case of the exciton $\mathrm{C}$. It is estimated that the characteristic time of $\mathrm{B}$ to $\mathrm{A}$ relaxation is $t_{\mathrm{BA}}=1 \mathrm{ps}$ and the lifetime of exciton $\mathrm{C}$ can be estimated as $\tau_{\mathrm{C}}=0.2 \mathrm{ps}$.

This research was partially supported by the Committee for Scientific Research (Poland) grant 7 T08A 06110.

\section{References}

[1] R. Stępniewski, K.P. Korona, A. Wysmołek, J.M. Baranowski, K. Pakuła, M. Potemski, G. Martinez, I. Grzegory, S. Porowski, to be published.

[2] I. Broser, M. Rosenzweig, R. Broser, M. Richard, E. Birkicht, Phys. Status Solidi $B$ 90, 77 (1978); M. Rosenzweig, Phys. Status Solidi B 129, 187 (1985).

[3] S. Nakajima, Y. Toyozawa, R. Abe, The Physics of Elementary Excitations, Springer-Verlag, New York 1980.

[4] R.J. Elliott, Phys. Rev. 108, 1384 (1957).

[5] S.I. Pekar, J. Phys. Chem. Solids 5, 11 (1958).

[6] G. Blattner, G. Kurtze, G. Schmieder, C. Klingshirn, Phys. Rev. B 25, 7413 (1982). 